\title{
Editorial for rare metals, special issue on solid state batteries
}

\author{
Hai-Jun Yu*, Yan Yu, Shu-Bin Yang
}

Published online: 14 May 2018

(C) The Nonferrous Metals Society of China and Springer-Verlag GmbH Germany, part of Springer Nature 2018

Energy storage devices based on organic liquid electrolyte are still suffered from safety issues. Therefore, developing the solid state batteries based on solid electrolytes is an inevitable choice because of the high safety. However, the practical applications of solid state batteries have been still suffered from some drawbacks, including the low ionic conductivity at room temperature, narrow electrochemical window, and weak chemical/electrochemical stability of solid-state electrolytes, the dendrite on metal anodes, and poor interfacial compatibility between electrodes and electrolytes.

This special issue consists of nine original research articles and two review articles on the research of solid state batteries. In detail, it covers the design of polymer electrolytes and inorganic solid electrolytes. Also, the composite metal anodes and the interfacial modifications between anodes and solid electrolytes have been deeply discussed. Furthermore, the energy storage devices applications, such as solid-state lithium batteries, sodium batteries, lithium-oxygen batteries, lithium-air batteries, lithium- $\mathrm{CO}_{2}$ batteries, and supercapacitors, are also included.

To date, there are mainly two key points that still to be solved for developing the solid state batteries: one is

\section{H.-J. Yu*}

College of Materials Science and Engineering, Beijing

University of Technology, Beijing 100124, China

e-mail: hj-yu@bjut.edu.cn

Y. Yu

Department of Materials Science and Engineering, University of

Science and Technology of China, Hefei 230026, China

\section{S.-B. Yang}

School of Materials Science and Engineering, Beihang

University, Beijing 100191, China fabricating solid-state electrolytes with high ionic conductivities at room temperature, and the other is how to construct the high-performance solid state batteries with intimate interfaces between solid electrolytes and electrodes. Aiming to these, new types of solid-state electrolytes, new methods for fabricating the electrolytes, and new techniques for building the solid state batteries are further to be explored. This journal will continue to offer more opportunities for publishing high-quality and highimpact papers in the research of solid state batteries.

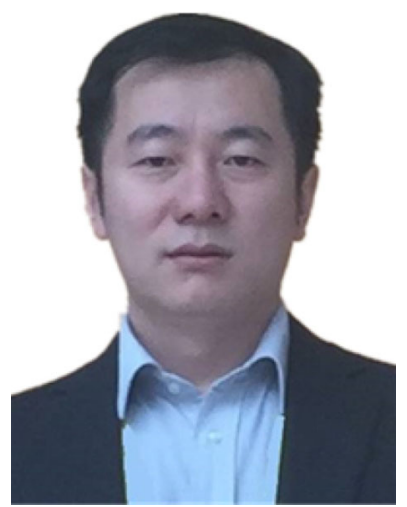

Hai-Jun Yu is currently a Professor of Materials Science in Beijing University of Technology. He received his Ph.D. in 2007 from Northeastern University, China. During the period (2007-2010) at General Research Institute for Nonferrous Metals, he was working on the Ni-MH power battery and EV application. After that, from 2010 to 2015 , he did research works on advanced battery materials at National Institute of Advanced Industrial Science and Technology in Japan. His research interests involve in the advanced electrode materials and devices for energy storage systems. Until now, he has published over 70 papers in peer-reviewed journals and filed 30 patents in the field of new energy materials. He was also awarded as "National Science Fund for Outstanding Young Scholars" and "Thousand Youth Talents Plan of China" in 2016. 


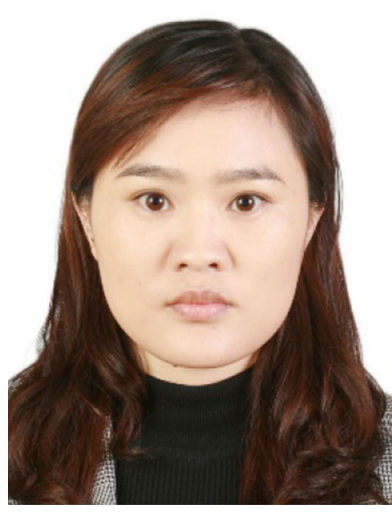

Yan Yu is a Professor of Materials Science in University of Science and Technology of China (USTC). She received her Ph.D. in Materials Science at USTC in 2006. From 2007 to 2008 , she worked as a postdoctoral at Florida International University. After that she received Humboldt Research Fellow and the Sofja Kovalevskaja award from the Alexander von Humboldt Foundation and worked at the Max Planck Institute for SolidState Research in Stuttgart, Germany. Her current research interests mainly include design of novel nanomaterials for clean energy, especially for batteries and the fundamental science of energy storage system. She has published more than 140 SCI papers.

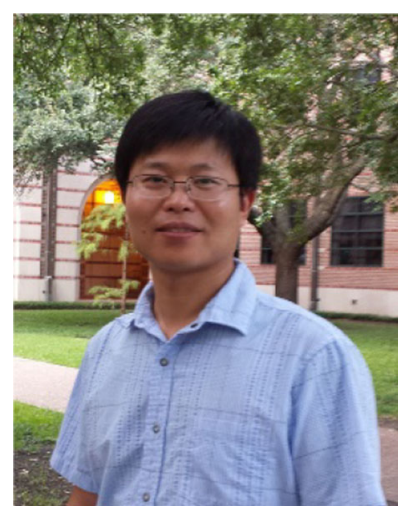

Shu-Bin Yang is currently a Full Professor at School of Materials Science and Engineering, Beihang University, since 2014. He received his $\mathrm{Ph} . \mathrm{D}$. from Beijing University of Chemical Technology in 2008. And then, he pursued postdoctoral research with Prof. K. Muellen and P.M. Ajayan at Max Planck Institute for Polymer Research and Rice University, respectively. His current research interests involve in graphene, MXenes, and other two-dimensional materials for energy storage and conversions. Until now, he has published over 70 papers in peer-reviewed journals including Adv. Mater., Angew. Chem. Int. Ed., Energy and Environ. Sci., etc., in the field of new energy materials. He was also awarded as "Thousand Youth Talents Plan of China" in 2014, "National Science Fund for Outstanding Young Scholars" in 2016, and Clarivate Analytics "Highly Cited Researchers" in 2017. 